\title{
A Fiber Optic Vertical Coupler for the Matrix Tactile Sensor
}

\author{
Kentaro Nakamura, Member and Masaru Uchiyama, Non-member \\ (Precision and Intelligence Laboratory, Tokyo Institute of Technology)
}

\begin{abstract}
This study proposes an optical fiber coupler sensitive to strain for matrix-style sensor array. It consists of a crossed fiber pair which has optical coupling through the radiated light from the grooves made on the fibers. The stress/strain is detected as a modulation of the magnitude of optical coupling. In this letter, this optical coupler has been applied to a 4 x 4 sensor array for trial, and some measurements of stress distribution are demonstrated.
\end{abstract}

Keywords: Tactile Sensor, Fiber Optic Coupler, Matrix-style Readout

\section{INTRODUCTION}

A device for multi-point sensing of stress/touch is highly required in robotics and other industries. A fiber optic tactile array sensor has been proposed, where matrix-style integration of sensors has been introduced[1]. In that work, a fiber with abrasion was used to produce the optical coupling. This letter proposes a new element sensor usable for this purpose. The proposed element sensor consists of a crossed fiber pair which has small grooves on fibers. The use of groove has provided the efficient and controllable optical coupling. This element sensor is integrated to a $4 \times 4$ matrix tactile sensor array, and applied to some measurements of distributed load. The matrixstyle configuration and the time-division readout by switching light sources gives efficient data acquisition[2].

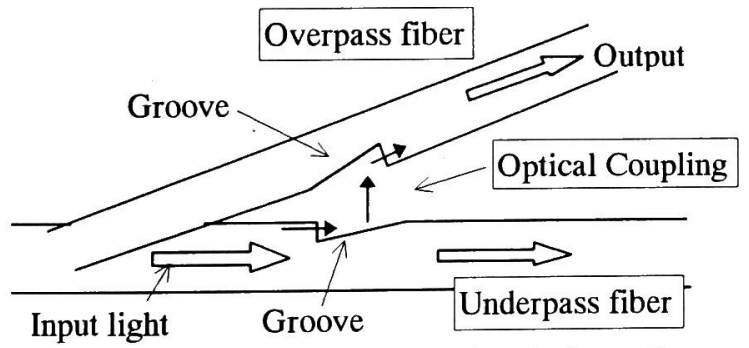

Fig. 1 Configuration of the vertical optical coupling.

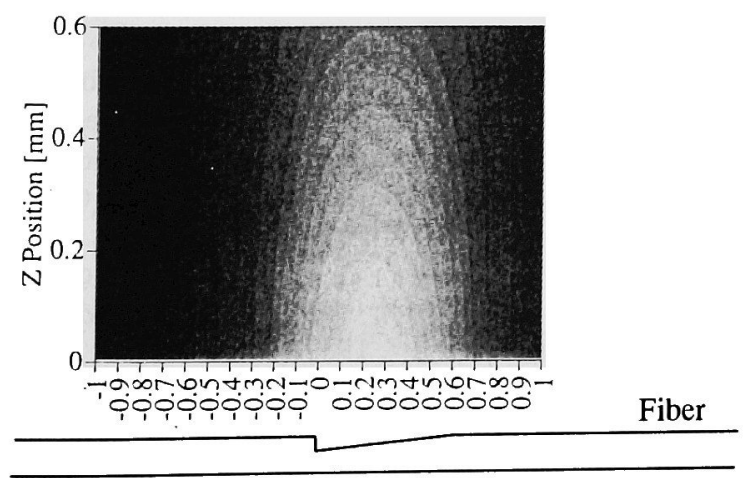

Fig.2 Spatial distribution of the light intensity radiated from the groove on fiber.

\section{VERTICAL COUPLER STRESS SENSOR}

Figure 1 shows the optical fiber vertical coupler developed for stress sensing. Two multimode plastic optical fibers are crossed as like in a grade separation. A small asymmetric groove is made on the fiber to radiate a part of transmitted light into the vertical direction to the fiber axis. The light goes out from the vertical wall of the groove and is reflected by the sloped wall of the groove. The intensity distribution of the radiated light was measured as shown in Fig. 2, where a bare optical fiber was used as an optical pick-up. Here, the plastic fiber of $2 \mathrm{~mm}$ in diameter was used, and the groove is $0.5 \mathrm{~mm}$ deep and $1 \mathrm{~mm}$ long. The radiated light is received by the overpass fiber which has the same groove. The distance between the two fibers can be measured by the output light intensity of the overpass fiber. The light of the underpass fiber transmitted through the groove is affected little by the displacement. If an appropriate elastic material is inserted between the fibers, the vertical load to the overpass fiber can be measured.

\section{MATRIX-STYLE INTEGRATION}

Figure 3 illustrates the $4 \times 4$ integrated sensor and the configuration of the element sensor. The underpass fiber lies inside the ditch of silicone viscoelastic pad, while the overpass fiber is adhered on the back of an aluminum plate of $15 \mathrm{~mm}$ square. The spacing between the fibers is adjusted by the thickness of the silicone pad. The optical coupling between the fibers at one element was measured as a function of the load applied on the aluminum plate as shown in Fig. 4. It is observed that the output is changed monotonously by the load, while the transmitted light intensity is almost constant. The sensitivity to the stress, i.e. the slope of curve in Fig.4, can be controlled by the elastic property or the thickness of the silicone pad.

Then, sixteen element sensors are connected together in matrix as illustrated in the figure. Every underpass fiber has a light emitting diode, and every overpass fiber is terminated by a photo detector. Plastic optical fiber of 1 $\mathrm{mm}$ in diameter with the numerical aperture of 0.5 was 


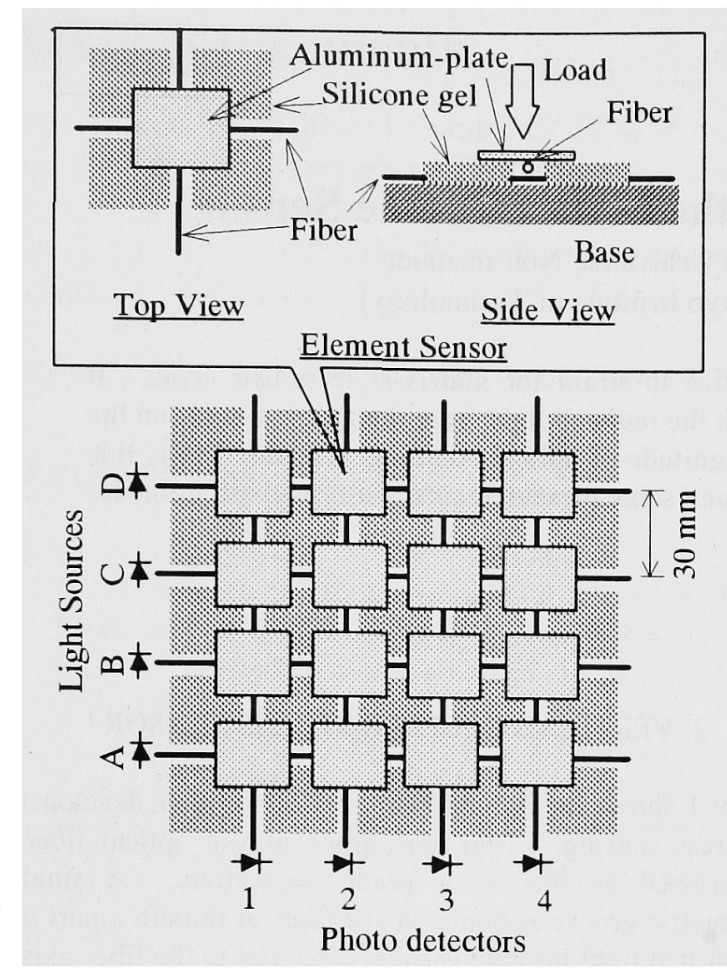

Fig.3 Structure of the $4 \times 4$ array sensor.

used. The groove is $0.2 \mathrm{~mm}$ in depth and $0.5 \mathrm{~mm}$ in length. If the light source \#B is turned on, the magnitude of optical coupling at the element sensors from $(B, 1)$ to $(B, 4)$ are read by the photodetectors from \#1 to \#4, respectively. If the light source is switched to \#C from \#B, the information at from $(C, 1)$ to $(C, 4)$ is acquired by the photodetector array. The high speed switching of light sources in this way gives a matrix-style readout of the distributed load.

Two examples of the stress distribution measurement are demonstrated in Fig. 5 by the prototype sensor. The diameter of solid circle in the figure shows the magnitude of load. First, a $1 \mathrm{~kg}$ weight was put over the element (A,2), $(A, 3),(B, 2)$ and $(B, 3)$. The position and the quantity of the stress can be sensed successfully, though small ghost signals are detected at $(\mathrm{C}, 1)$ and $(\mathrm{C}, 3)$. Next one is a demonstration for two $1 \mathrm{~kg}$ weights: one weight was put on $(A, 1),(A, 2),(B, 1)$ and $(B, 2)$, and the other one on $(B, 3)$, $(B, 4),(C, 3)$ and $(C, 4)$. Element sensors are mechanically connected each other, and too much stress may cause a mechanical interaction with the adjacent elements and produce the ghost signal.

\section{SUMMARY}

A new fiber coupler suitable for matrix-style distributed stress sensor has been developed, and a $4 \times 4$ twodimensional sensor was made for trial. The sensitivity for stress can be controlled by the hardness of silicone insulating gel. The measurements of stress distribution were demonstrated successfully by the prototype sensor.

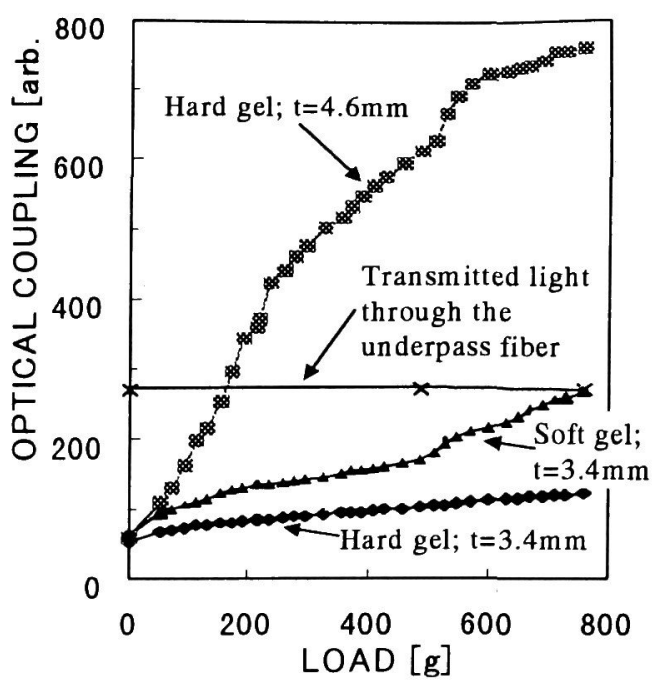

Fig.4 Optical coupling vs. the load.
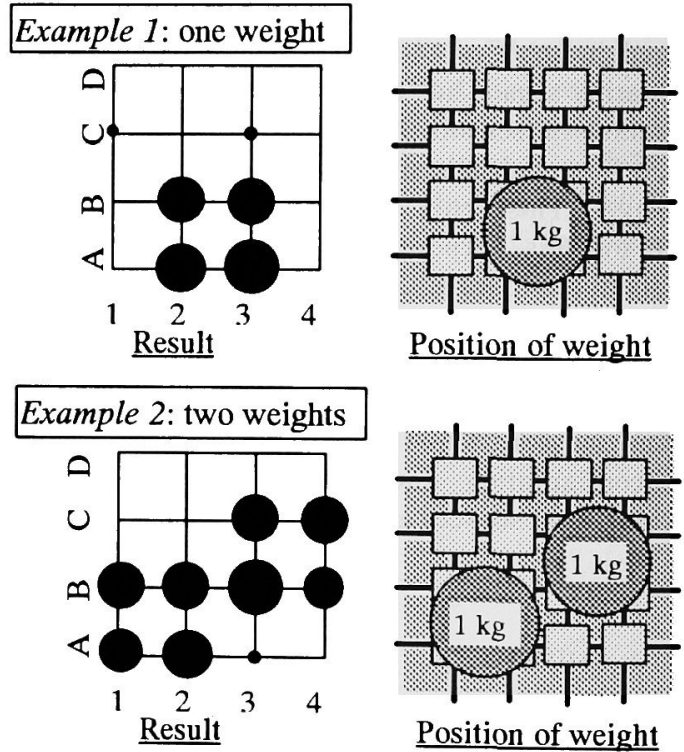

Fig.5 Stress distributions measured by the proposed sensor. Solid circles indicate the magnitude.

Miniaturization and utilization of polymer sheet waveguide should be made in the future study.

\section{ACKNOWLEDGEMENT}

This study was partly supported by Grant-in-Aid for COE Research from the Ministry of Education, Science, Sport and Culture (\#07CE2003, "Ultra-parallel Opto-electronics")

(Manuscript received June 26, 1998, revised Oct. 6, 1998) REFERENCES

[1]J. S. Schoenwald et al., "A novel fiber optic array sensor," Proc. IEEE Int. Conf. on Robotics and Automation, pp.1792-1797, 1987.

[2]K. Nakamura and K. Fukaya, "Optical fiber coupler array for multi-point sound field measurements," Optical Review, Vol.4, No.1A, 65-68, Jan., 1997. 